\title{
Aspectos epidemiológicos da tuberculose em menores de 15 anos no Município de São Paulo, Brasil, 1984*.
}

\section{Epidemiological aspects of tuberculosis for minors under 15 years old in a County of the Brazilian Southeast Region, 1984.}

\author{
Marialda Höfling de Pádua Dias **
}

DIAS, M.H. de P. Aspectos epidemiológicos da tuberculose em menores de 15 anos no Município de São Paulo, Brasil, 1984. Rev. Saúde públ., S. Paulo, 25: 426-34, 1991. Foram estudados todos os casos de tuberculose, referentes a 1984, envolvendo população residente no Município de São Paulo, SP, Brasil, na faixa etária de menores de 15 anos, cujo diagnóstico foi notificado ao Centro de Informações da Saúde da Secretaria de Estado da Saúde (SP). Foram analisados os prontuários em cada instituição que fez a notificação e realizadas entrevistas com os médicos responsáveis pelos casos. A incidência foi de $21,4 / 100.000$ nos menores de 15 anos, a maior ocorrendo no grupo etário de menores de 5 anos (31,8/100.000 habitantes). Foram encontradas grandes diferenças entre os coeficientes correspondentes às diversas regiões do Município. A forma de tuberculose mais freqüente foi a pulmonar (83,1\%-17,8/100.000) enquanto que a extrapulmonar foi de $12,2 \%(2,5 / 100.000)$ e a pulmonar associada a outras localizações extrapulmonares, de 4,7\% (1,0/100.000 habitantes). Foram encontrados 46 casos com BK + correspondendo a $37,7 \%$ dos exames realizados, em material de origem pulmonar, e 16 casos com $\mathrm{BK}+(53,3 \%$ dos exames realizados $)$, em material extrapulmonar. $\mathrm{O}$ coeficiente de incidência de casos com baciloscopia positiva de escarro foi de $0,9 / 100.000$, sendo maior na idade de 10 a 14 anos $(2,8 / 100.000)$.

Descritores: Tuberculose infantil, epidemiologia. Notificação de doenças.

\section{Introdução}

Antes da era dos quimioterápicos, a mortalidade foi um indicador muito útil na avaliação da situação da tuberculose ${ }^{30.38}$. No entanto, a partir da introdução da quimioterapia, passou a ser mais útil na avaliação da eficiênuia dos serviços de saúde do que para verificar a situação epidemiológica da tuberculose, pois a eficácia dos medicamentos reduziu a mortalidade a níveis muito baixos ${ }^{30,38}$.

Por outro lado, o risco de um indivíduo se infectar, no período de um ano, considerado o indicador mais fidedigno na avaliação da tuberculose em uma determinada região ${ }^{28.30 .34}$, não pode ser calculado com base em iniquéritos de prevalência de infecção tuberculosa, como geralmente é realiza-

* Parte de tese de Doutorado subordinada ao mesmo título, apresentada à Faculdade de Saúde Pública da Universidade de São Paulo, 1989

* Instituto da Criança do Hospital das Clínicas da Faculdade de Medicina da USP. São Paulo, SP Brasil

Separatas/Reprints: M.H. de P. Dias - Av. Dr. Enéas Carvalho de Aguiar, 647 - 05403 - São Paulo, SP Brasil

Publicação financiada pela FAPESP. Processo Medicina 90/4602-1 $\mathrm{do}^{1,32,34}$, visto que a cobertura de BCG no Brasil, já em 1981, estava muito acima do limite indicado pela Organização Mundial da Saúde (OMS) para avaliar a prevalência da infecção, sem que vícios possam ocorrer ${ }^{37}$.

Por essas considerações, expostas acima, a taxa de morbidade é empregada para avaliar a situação epidemiológica da tuberculose, utilizando-se, para esse fim, a notificação de casos novos de tuberculose, principalmente no que se refere a casos bacilifferos. Styblo e Sutherland ${ }^{30}$ fazem críticas a esse indicador, salientando a existência de subnotificação e a falta de uniformidade da definição de "caso novo", onde se incluem casos positivos à cultura, casos negativos ao exame bacteriológico e casos não examinados bacteriologicamente. Styblo e Rouillon ${ }^{29}$ referem-se também à não confiabilidade das cifras de morbidade, o mesmo ocorrendo com Bulla ${ }^{45}$.

Em países desenvolvidos o sistema de notificação tem sofrido críticas, como aquela referida por Davies e col. ${ }^{11} \mathrm{em}$ relação à pesquisa realizada pelo Medical Research Council Tuberculosis and Chest Diseases $^{22}$.

Mesmo se sabendo que existem críticas à taxa de casos novos de tuberculose notificados, e mesmo se sabendo que a incidência encontrada no Brasil é sempre uma parcela da real ${ }^{9}$, $(75 \%$ aproximadamente da taxa esperada) o estudo da morbidade da 
tuberculose, baseada na notificação de casos novos, é um indicador operacionalizável no momento.

Por outro lado, como referem Styblo e Sutherland $^{31}$, também há falta de informações sobre a situação da tuberculose da criança.

Com a finalidade de contribuir para o conhecimento da situação epidemiológica da tuberculose em menores de 15 anos, o presente trabalho foi realizado com o objetivo de descrever algumas características dos casos notificados, no que se refere à idade, sexo, local de residência, forma de tuberculose, localização da doença segundo órgãos ou sistemas e positividade à pesquisa de bacilo de Koch (BK).

\section{Metodologia}

A pesquisa foi desenvolvida no Município de São Paulo, capital do Estado de São Paulo, que, em 1984 possuia uma população de 9.432 .626 habitantes, dos quais 3.062.697 eram menores de 15 anos. A área do Município, de $1.508,99 \mathrm{~km}^{2}$, estava dividida em 9 zonas e 56 distritos ou subdistritos de $\mathrm{Paz}^{33}$.

Foram estudados todos os casos de tuberculose em menores de 15 anos, residentes no Município de São Paulo, notificados pela primeira vez ao Centro de Informações da Saúde (CIS) e referentes ao ano de 1984.

No CIS foram levantadas 849 fichas de notificação, consultando-se a seguir os prontuários, nas instituições onde se encontravam. Foram excluídos 45 casos por notificação indevida face ao reconhecimento pelo próprio médico de que não eram casos de tuberculose, 45 por idade informada erroneamente como sendo menor de 15 anos e 19 casos cujo ano de diagnóstico era diferente de 1984. Dos 740 casos restantes, foram retirados 5 , cujos prontuários não foram encontrados e 79 não residentes no Município de São Paulo, ficando o número de casos analisados reduzido para 656.
Os dados foram obtidos das anotações dos prontuários e complementados pelos médicos que notificaram os casos. Assim, foram anotadas em questionário as características dos casos notificados referentes, entre outras, à idade, sexo, forma de tuberculose, localização da doença em órgãos ou sistemas e resultado da pesquisa de BK.

Os critérios diagnósticos utilizados pelos médicos, objeto de análise crítica em futuro trabalho, não sofreram interferência da pesquisa. As técnicas utilizadas para a realização de exames complementares foram aquelas em uso nas respectivas instituições.

A coleta de dados foi feita pela autora, no ano de 1985, tendo sido concluída em fevereiro de 1986. Foram visitadas 55 instituições e dos 103 médicos que notificaram os casos, três não foram encontrados. Dessa forma, sete casos não foram vistos com os médicos.

Os dados foram processados em microcomputador.

\section{Resultados}

$O$ perfil da tuberculose em menores de 15 anos pode ser observado na Tabela 1 , onde se nota que a faixa etária de menores de 5 anos é a que apresenta maior proporção $(58,1 \%)$ de casos, seguindo-se a de 5 a $9(25,3 \%)$ e a de 10 a 14 anos $(16,6 \%)$. O mesmo fato pode ser verificado em relação ao coeficiente de incidência, 31,8; 15,9 e 13,3/100.000 habitantes, respectivamente, em menores de 5 anos, no grupo de 5 a 9 e 10 a 14 anos, com um total de $21,4 / 100.000$ em menores de 15 anos.

A distribuição, segundo o sexo, colocada na mesma Tabela, mostra que há pequena diferença entre os sexos, tanto percentual como quanto ao coeficiente de incidência, $51,7 \% / 48,3 \%$ e $21,9 / 20,9 /$ 100.000 , respectivamente para o sexo masculino e feminino, permanecendo o perfil decrescente nos três grupos etários, em ambos os sexos.

Tabela 1. Casos de tuberculose notificados em menores de 15 anos, segundo sexo e faixa etária ${\text { ( }{ }^{\circledR}}^{2} 20$ coeficiente/ 100.000 habitantes). Município de São Paulo - 1984

\begin{tabular}{|c|c|c|c|c|c|c|c|c|c|c|c|c|}
\hline \multirow{2}{*}{$\begin{array}{l}\text { Sexo } \\
\text { Faixa } \\
\text { Etária (anos) }\end{array}$} & \multicolumn{4}{|c|}{ Masculino } & \multicolumn{4}{|c|}{ Feminino } & \multicolumn{4}{|c|}{ Total } \\
\hline & $n^{0}$ & $\%$ & Popul. & Coef. & $n^{2}$ & $\%$ & Popul. & Coef. & № & $\%$ & Popul. & Coef. \\
\hline $0 a 4$ & 205 & $\begin{array}{c}60,5 \\
(53,8)\end{array}$ & 610342 & 33,6 & 176 & $\begin{array}{c}55,5 \\
(46,2)\end{array}$ & 589177 & 29,9 & 381 & $\begin{array}{c}58,1 \\
(100,0)\end{array}$ & 1199519 & 31,8 \\
\hline 5 a 9 & 76 & $\begin{array}{c}22,4 \\
(45,8)\end{array}$ & 527051 & 14,4 & 90 & $\begin{array}{c}28,4 \\
(54,2)\end{array}$ & 514200 & 17,5 & 166 & $\begin{array}{c}25,3 \\
(100,0)\end{array}$ & 1041251 & 15,9 \\
\hline 10 a 14 & 58 & $\begin{array}{c}17,1 \\
(53,2)\end{array}$ & 412004 & 14,1 & 51 & $\begin{array}{c}16,1 \\
(46,8)\end{array}$ & 409923 & 12,4 & 109 & $\begin{array}{c}16,6 \\
(100,0)\end{array}$ & 821927 & 13,3 \\
\hline Total & 339 & $\begin{array}{l}100,0 \\
(51,7)\end{array}$ & 1549397 & 21,9 & 317 & $\begin{array}{l}100,0 \\
(48,3)\end{array}$ & 1513300 & 20,9 & 656 & $\begin{array}{c}100,0 \\
(100,0)\end{array}$ & 3062697 & 21,4 \\
\hline
\end{tabular}

() Valor entre parênteses representa percentagem calculada na linha. 


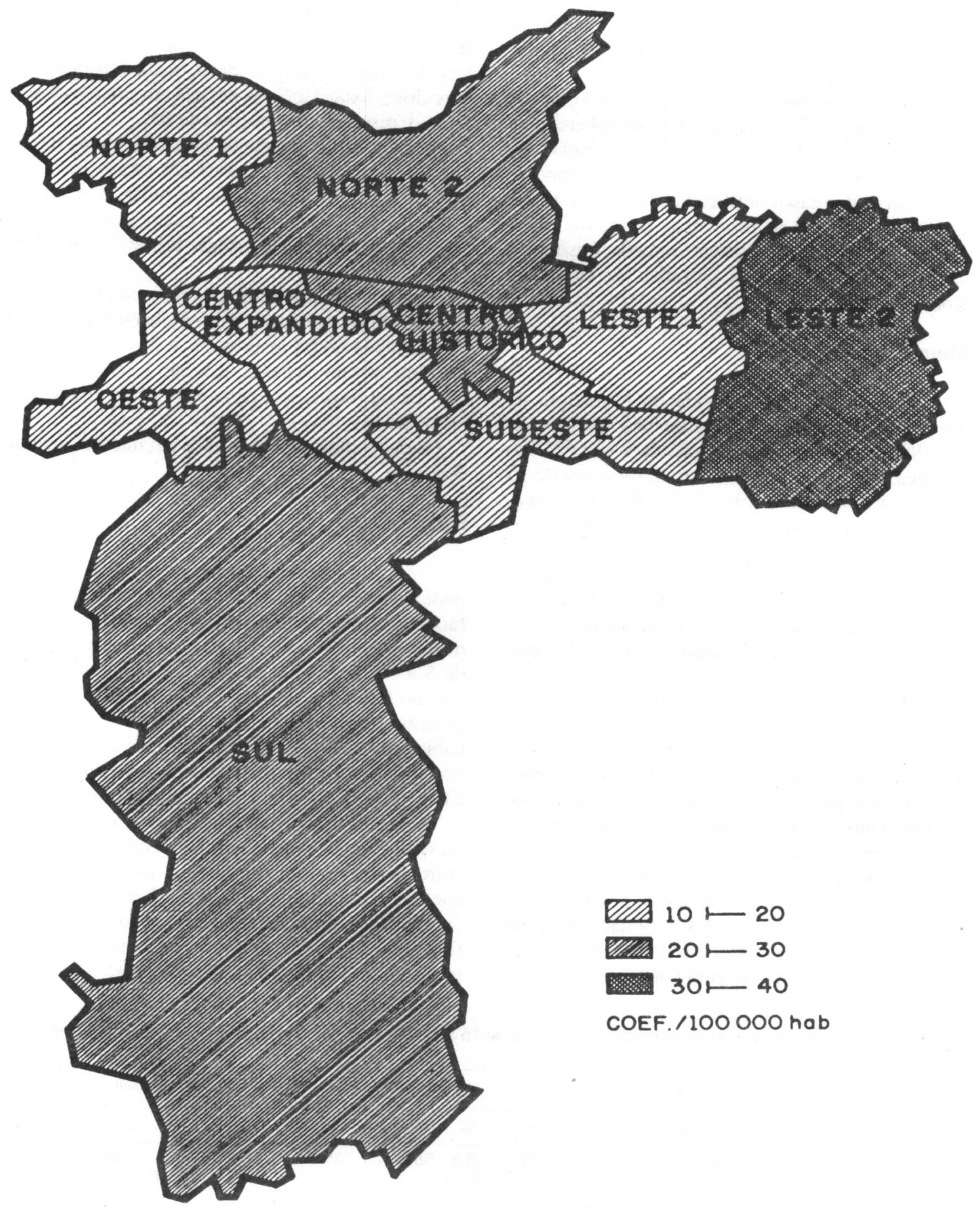
Figura. Incidência de casos de tuberculose notificados em menores de 15 anos, segundo zonas de residência.
Município de São Paulo - 1984 . 
A distribuição dos casos, segundo a região de residência, está colocada na Tabela 2 , sendo melhor visualizada na Figura.

Nota-se que a Zona Leste 2 apresentou o maior coeficiente de incidência $(33,6 / 100.000)$, seguindose a Zona Sul $(26,4 / 100.000)$. Centro Histórico $(26,2)$, Zona Norte $2(22,0 / 100.000)$. As outras zonas apresentaram coeficientes menores de 20/ 100.000 habitantes.

Quanto à forma, a tuberculose foi classificada em pulmonar, extrapulmonar e associada, considerando-se pulmonar, quando a localização é pulmonar (localizada ou miliar) e associada, quando há associação entre a localização pulmonar (inclusive a miliar) e extrapulmonar (Tabela 3).

Observa-se, na Tabela 3 , a predominância da forma pulmonar $(83,1 \%)$, seguindo-se a extrapulmonar $(12,2 \%)$ e associada $(4,7 \%)$. Em relação aos coeficientes, a distribuição foi de 17,8 , 2,6 e 1,0/100.000, respectivamente.

Verifica-se também, nos grupos etários, um perfil decrescente na forma pulmonar, sendo diferente na extrapulmonar onde o grupo de maior incidência foi o de 10 a 14 anos e de 5 a 9 anos (respectivamente 3,0 e $2,9 / 100.000$ ).

$\mathrm{Na}$ forma associada, o grupo de mainr incidên-

Tabela 2. Casos de Tuberculose notificados em menores de 15 anos, segundo Zona de Residência (№, \% População e coeficiente $/ 100.000$ Habitantes) Município de São Paulo - 1984.

\begin{tabular}{lrrcc}
\hline Zona & $\mathrm{N}^{2}$ & $\%$ & População* & Coeficiente \\
\hline Centro Histórico & 19 & 3,0 & 72.607 & 26,2 \\
Centro Expandido & 30 & 4,6 & 261.181 & 11,5 \\
Oeste & 21 & 3,2 & 124.409 & 16,9 \\
Sul & 134 & 28,0 & 701.401 & 26,4 \\
Sudeste & 39 & 5,6 & 362.510 & 10,8 \\
Leste 1 & 65 & 9,8 & 401.214 & 16,2 \\
Leste 2 & 158 & 24,0 & 470.924 & 33,6 \\
Norte 1 & 16 & 2,5 & 114.555 & 13,9 \\
Norte 2 & 122 & 18,6 & 553.896 & 22,0 \\
Indeterminada & 2 & 0,3 & & \\
Município de & & & & \\
São Paulo & 656 & 100,0 & 3.062 .697 & 21,4 \\
\hline
\end{tabular}

*Fonte: Fundação SEADE cia foi aquele de menores de 5 anos $(1,9 / 100.000)$, seguindo-se o de 10 a 14 e de 5 a 9 anos $(0,5$ e $0,4 /$ 100.000 respectivamente).

A localização da tuberculose em órgãos ou sistemas pode ser vista na Tabela 4 , onde se nota que a pulmonar isolada, excluindo-se a miliar, corresponde a $80,6 \%$, ou seja, uma incidência de 17,3/100.000 habitantes. Observando-se os mesmos dados independentemente da localização ser isolada ou associada (Tabela 5), a distribuição é um pouco diferente $(84,1 \%)$, com um coeficiente de $18 /$ 100.000 habitantes, para a localização pulmonar. A meningite, por sua vez, foi responsável por um coeficiente de $1 / 100.000$, a pleural 0,9 , miliar 0,8 , a ganglionar periférica 0,7 ; renal 0,4 , osteoarticular 0,4 , ocular 0,1 , peritonial 0,03 e de partes moles $0,03 / 100.000$ habitantes.

Nessa Tabela 5 estão referidas as percentagens e coeficientes referentes à localização da doença, segundo os grupos etários. Nota-se que, para a localização pulmonar, o coeficiente é de 28,0/100.000 no grupo de menores de 5 anos, de 13,0/100.000 no de 5 a 9 anos e $9,8 / 100.000$ no de 10 a 14 anos. No grupo etário de menores de 5 anos o segundo maior coeficiente encontrado foi o de meningite $(1,8 /$ $100.000)$, seguindo-se o de tuberculose miliar $(1,7 /$ 100.000 ). No grupo de 5 a 9 anos o segundo maior coeficiente foi o de meningite $(0,8 / 100.000)$, seguindo-se o de tuberculose renal e ganblionar periférica $(0,7 / 100.000)$. No grupo de 10 a 14 anos o segundo maior coeficiente ocorreu na localização pleural $(1,8 /$ 100.000).

A Tabela 6, por sua vez, mostra a realização do exame bacteriológico, segundo o material utilizado, independente da forma de tuberculose. Verifica-se que o número de exames realizados, em material pulmonar, foi de $22,4 \%$ do total dos casos em que haveria indicação para fazê-lo, enquanto que em material extrapulmonar foi de $37,5 \%$ dos casos. O número de casos com pesquisa positiva para $\mathrm{BK}$, em material pulmonar, foi de 46 , ou seja, $35,7 \%$ dos exames realizados, englobando tanto o exame bacterioscópico de escarro ou de lavado gástrico como a cultura do mesmo material. Em material

Tabela 3. Casos de tuberculose notificados em menores de 15 anos, segundo faixa etária e forma de tuberculose ( $n^{\circ}, \%$ e Coeficiente/100.000 habitantes). Município de São Paulo, 1984.

\begin{tabular}{|c|c|c|c|c|c|c|c|c|c|c|c|c|}
\hline \multicolumn{2}{|c|}{$\begin{array}{c}\text { Forma de } \\
\text { Tuberculose }\end{array}$} & \multicolumn{2}{|c|}{ Pulmonar } & \multicolumn{3}{|c|}{ Extrapulmonar } & \multicolumn{3}{|c|}{ Associada } & \multicolumn{3}{|c|}{ Todas as formas } \\
\hline etária (anos) & $n^{2}$ & $\%$ & Coef. & $n^{8}$ & $\%$ & Coef. & $\mathrm{n}^{\circ}$ & $\%$ & Coef. & $n^{2}$ & $\%$ & Coef \\
\hline 0 a 4 & 333 & 61,1 & 27,8 & 25 & 31,3 & 2,1 & 23 & 74,2 & 1,9 & 381 & 58,1 & 31,8 \\
\hline 5 a 9 & 132 & 24,2 & 12,7 & 30 & 37,4 & 2,9 & 4 & 12,9 & 0,4 & 166 & 25,3 & 15,9 \\
\hline 10 a 14 & 80 & 14,7 & 9,7 & 25 & 31,3 & 3,0 & 4 & 12,9 & 0,5 & 109 & 16,6 & 13,3 \\
\hline Total & 545 & $\begin{array}{l}100,0 \\
(83,1)\end{array}$ & 17,8 & 80 & $\begin{array}{r}100,0 \\
(12,2)\end{array}$ & 2,6 & 31 & $\begin{array}{c}100,0 \\
(4,7)\end{array}$ & 1,0 & 656 & $\begin{array}{c}100,0 \\
(100,0)\end{array}$ & 21,4 \\
\hline
\end{tabular}


Tabela 4. Casos de tuberculose notificados em menores de 15 anos, segundo localização da doença em órgãos e/ ou sistemas ( $n^{\circ}, \%, \operatorname{coef} / 100.000$ habitantes) Municipio de São Paulo - 1984.

\begin{tabular}{lrrr}
\hline Localização da Tuberculose & $n^{0}$ & $\%$ & coef. \\
\hline Pulmonar & 529 & 80,6 & 17,3 \\
Pleural & 19 & 2,9 & 0,6 \\
Meningite & 15 & 2,3 & 0,5 \\
Miliar & 16 & 2,4 & 0,5 \\
Ganglionar Periférica & 15 & 2,3 & 0,5 \\
Renal & 12 & 1,8 & 0,4 \\
Osteoarticular & 12 & 1,8 & 0,4 \\
Ocular & 4 & 0,6 & 0,1 \\
Peritonial & 1 & 0,2 & $(0,03)^{*}$ \\
Partes Moles & 1 & 0,2 & $(0,03)^{*}$ \\
Pulmonar + Meningite & 8 & 1,2 & 0,3 \\
Miliar + Meningite & 8 & 1,2 & 0,3 \\
Pulmonar + Pleural & 10 & 1,5 & 0,3 \\
Pulmonar + Gangl. Periférica & 5 & 0,8 & 0,2 \\
Pleural + Meningite & 1 & 0,2 & $(0,03)^{*}$ \\
\hline Total & 656 & 100,0 & 21,4
\end{tabular}

* Calculado até a $2^{\text {a }}$ casa decimal para nāo apresentar o coeficiente como nulo. extrapulmonar, 16 casos apresentaram $\mathrm{BK}$ positivo ou seja $53,3 \%$ do total de exames realizados.

$\mathrm{Na}$ Tabela 7 estão colocados somente os casos positivos à pesquisa de $\mathrm{BK}$, em material pulmonar, juntamente com os respectivos coeficientes distribuídos por faixa etária. $\mathrm{O}$ coeficiente mais importante do ponto de vista epidemiológico, o de baciloscopia positiva ao exame de escarro, foi de $0,9 / 100.000$ habitantes em menores de 15 anos, sendo zero no grupo de menores de 5 anos, de $0,4 /$ 100.000 , no de 5 a 9 anos e de $2,8 / 100.000$, no de 10 a 14 anos.

\section{Discussão}

A distribuição dos casos de tuberculose, no $\mathrm{Mu}$ nicípio de São Paulo, repete o perfil da tuberculose no Estado de São Paulo* no mesmo ano $(58,5 \%$ em menores de 5 anos, 23,4\% no grupo etário de 5 a 9 anos e $18,1 \%$ no de 10 a 14 anos). O mesmo perfil decrescente, para os grupos etários, ocorreu também

Tabela 5. Casos de tuberculose notificados em menores de 15 anos, segundo faixa etária e localização da doença, independente da forma isolada ou associada ( $n^{\circ}, \%$ e coeficiente/100.000 habitantes). Município de São Paulo - 1984.

\begin{tabular}{|c|c|c|c|c|c|c|c|c|c|c|c|c|}
\hline \multirow{2}{*}{$\begin{array}{c}\text { Faixa Etária } \\
\text { (anos) } \\
\text { Localização } \\
\end{array}$} & \multicolumn{3}{|c|}{0 a 4} & \multicolumn{3}{|c|}{5 a 9} & \multicolumn{3}{|c|}{10 a 14} & \multicolumn{3}{|c|}{ Total } \\
\hline & $n^{2 *}$ & $\%$ & coef." & $n^{9}$ & $\%$ & coef. & $n^{2}$ & $\%$ & coef. & $\mathrm{n}^{\mathrm{Q* \star}}$ & $\%$ & coef.** \\
\hline $\begin{array}{l}\text { Pulmonar } \\
\text { Meningite } \\
\text { Pleural } \\
\text { Miliar } \\
\text { Ganqlionar }\end{array}$ & $\begin{array}{r}336 \\
21 \\
9 \\
20\end{array}$ & $\begin{array}{r}88,2 \\
5,5 \\
2,4 \\
5,3\end{array}$ & $\begin{array}{r}28,0 \\
1,8 \\
0,7 \\
1,7\end{array}$ & $\begin{array}{r}135 \\
8 \\
5 \\
1\end{array}$ & $\begin{array}{r}81,3 \\
4,8 \\
3,0 \\
0,6\end{array}$ & $\begin{array}{r}13,0 \\
0,8 \\
0,5 \\
0,1\end{array}$ & $\begin{array}{r}81 \\
3 \\
16 \\
3\end{array}$ & $\begin{array}{r}74,0 \\
2,8 \\
15,0 \\
2,8\end{array}$ & $\begin{array}{l}9,8 \\
0,4 \\
1,8 \\
0,4\end{array}$ & $\begin{array}{r}552 \\
32 \\
30 \\
24\end{array}$ & $\begin{array}{r}84,1 \\
4,9 \\
4,6 \\
3,7\end{array}$ & $\begin{array}{r}18,0 \\
1,0 \\
0,9 \\
0,8\end{array}$ \\
\hline $\begin{array}{l}\text { Periférica } \\
\text { Osteoarticular } \\
\text { Renal } \\
\text { Ocular } \\
\text { Peritonial } \\
\text { Partes Moles }\end{array}$ & $\begin{array}{r}10 \\
8\end{array}$ & $\begin{array}{l}2,6 \\
2,1\end{array}$ & $\begin{array}{l}0,8 \\
0,6\end{array}$ & $\begin{array}{l}7 \\
4 \\
7 \\
1 \\
1 \\
1\end{array}$ & $\begin{array}{l}4,2 \\
2,4 \\
4,2 \\
0,6 \\
0,6 \\
0,6\end{array}$ & $\begin{array}{l}0,7 \\
0,4 \\
0,7 \\
0,1 \\
0,1 \\
0,1\end{array}$ & $\begin{array}{l}3 \\
- \\
5 \\
3\end{array}$ & $\begin{array}{r}2,8 \\
5 \\
4,5 \\
2,8\end{array}$ & $\begin{array}{r}0,4 \\
0,6 \\
0,4\end{array}$ & $\begin{array}{r}20 \\
12 \\
12 \\
4 \\
1 \\
1\end{array}$ & $\begin{array}{l}3,1 \\
1,8 \\
1,8 \\
0,6 \\
0,2 \\
0,2\end{array}$ & $\begin{array}{c}0,7 \\
0,4 \\
0,4 \\
0,1 \\
(0,03)^{\star \star \star} \\
(0,03)^{\star \star \star}\end{array}$ \\
\hline
\end{tabular}

* Proporçóes e coeficientes calculados em relação ao total de casos das respectivas faixas etárias.

* Proporçōes e coeficientes calculados em relação ao total de casos.

*** Calculado até a $2^{\mathrm{a}}$ casa decimal para não apresentar o coeficiente como nulo.

Tabela 6. Casos de tuberculose notificados em menores de 15 anos, segundo realização do exame bacteriológico e seu resultado, em material onde o exame foi realizado, independentemente da forma de tuberculose. Município de São Paulo - 1984

\begin{tabular}{|c|c|c|c|c|c|c|c|c|c|c|}
\hline \multirow{3}{*}{$\begin{array}{l}\text { Exame bacteriológico } \\
\text { Material }\end{array}$} & \multicolumn{6}{|c|}{ Realizado } & \multicolumn{2}{|c|}{ Não realizado } & \multicolumn{2}{|c|}{ Total } \\
\hline & \multicolumn{2}{|c|}{$\mathrm{BK}+$} & \multicolumn{2}{|c|}{ BK- } & \multicolumn{2}{|c|}{ Total } & \multirow[b]{2}{*}{$N^{2}$} & \multirow[b]{2}{*}{$\%$} & \multirow[b]{2}{*}{$N^{2}$} & \multirow[b]{2}{*}{$\%$} \\
\hline & $n^{2}$ & $\%$ & $\mathrm{~N}^{2}$ & $\%$ & $N^{2}$ & $\%$ & & & & \\
\hline Pulmonar & $46^{*}$ & $(35,7)^{\star \star}$ & 83 & $(64,3)$ & 129 & 22,4 & 447 & 77,6 & 576 & 100,0 \\
\hline Extrapulmonar & $16^{*}$ & $(53,3)$ & 94 & $(46,7)$ & 30 & 37,5 & 51 & 62,9 & 81 & 100,0 \\
\hline
\end{tabular}

* Um caso positivo no lavado gástrico e no líquor

** Números entre parênteses () representam as proporçōes calculadas em relação ao total de exames realizados.

\footnotetext{
* Calculado a partir de dados fornecidos pelo Centro de Informações da Saúde (CIS)
} 
Tabela 7. Casos de tuberculose com exame bacteriológico positivo em material pulmonar, notificados em menores de 15 anos, segundo tipo de exame realizado e faixa etária ( $n^{2}, \%$, coeficiente/100.000 habitantes). Município de São Paulo $-1984$.

\begin{tabular}{|c|c|c|c|c|c|c|c|c|c|c|}
\hline \multirow{2}{*}{\multicolumn{2}{|c|}{$\begin{array}{l}\text { Tipo de exame } \\
\text { bacteriológico } \\
\text { Faixa etária (anos) }\end{array}$}} & \multicolumn{3}{|c|}{ Baciloscopia* } & \multicolumn{3}{|c|}{$\begin{array}{l}\text { Baciloscopia } \\
\text { e/ou cultura }\end{array}$} & \multicolumn{3}{|c|}{$\begin{array}{c}\text { Baciloscopia } \\
\text { de escarro }\end{array}$} \\
\hline & & $n^{9}$ & $\%$ & coef. & $n^{2}$ & $\%$ & coef. & $n^{2}$ & $\%$ & coef. \\
\hline & a 4 & $13^{\star \star}$ & 30,2 & 1,1 & $15^{\star \star \star \star \star}$ & 32,6 & 1,2 & & & \\
\hline & a 9 & $6^{* * *}$ & 14,0 & 0,6 & $7^{\star \star * \star * *}$ & 15,2 & 0,7 & 4 & 14,8 & 0,4 \\
\hline & a 14 & 24 & 55,8 & 2,9 & 24 & 52,2 & 2,9 & 23 & 85,2 & 2,8 \\
\hline Total & & 43 & 100,0 & 1,4 & 46 & 100,0 & 1,5 & 27 & 100,0 & 0,9 \\
\hline * & \multicolumn{4}{|c|}{$\begin{array}{l}\text { Escarro ou lavado gástrico. } \\
9 \text { + à baciloscopia de lavado gástrico; } \\
4 \text { + à baciloscopia e à cultura em lavado } \\
\text { gástrico. } \\
4 \text { + no escarro; } \\
2 \text { + no lavado gástrico. }\end{array}$} & $\star \star \star \star * * *$ & \multicolumn{5}{|c|}{$\begin{array}{l}4 \text { + à baciloscopia e cultura de lavado } \\
\text { gástrico; } \\
2 \text { + somente à cultura de lavado gástrico. } \\
1 \text { + à cultura do lavado Ġíttico. }\end{array}$} \\
\hline
\end{tabular}

em Salvador ${ }^{7}$, em $1980(48,3 \%, 34,7 \%$ e $17,0 \%)$ e no Brasil*, em 1.984 (51,2\%, 24,7\% e $24,1 \%)$, respectivamente nos grupos etários considerados.

Essa distribuição é diferente quando comparada a de países como a Inglaterra ${ }^{22}$, onde o grupo de maior frequiência foi o de 5 a 9 anos, em trabalho realizado em 1978-1979. Em outro trabalho, no mesmo país, em $1983^{23}$, o grupo de maior frequiência foi o de 10 a 14 anos, sendo o segundo o de menores de 5 anos e o terceiro o de 5 a 9 anos.

O perfil da distribuição dos coeficientes é muito parecido com a do Estado de São Paulo **(31,3/ $100.000,14,0$ e $12,7 / 100.000$ ) para as três faixas etárias, de menores de 5 anos, 5 a 9 e 10 a 14 anos. No Brasil**, o mesmo coeficiente foi um pouco diferente $(24,6 ; 13,9$ e 14,2/100.000 habitantes respectivamente), nas três faixas etárias.

Quanto ao coeficiente total em menores de 15 anos, no Município de São Paulo - 21,4/100.0000 foi muito próximo ao do Estado de São Paulo** $(20,2 / 100.000)$ e maior que o Brasil nesse ano de $1984 * * *(17,8 / 100.000)$.

Os trabalhos realizados pelo Medical Research Council em 1978-1979 e em 1983 mostram coeficientes de $7 / 100.000$ e de $5,1 / 100.000$ respectivamente ${ }^{22,23}$. Na Suécia ${ }^{27}$, o coeficiente encontrado foi de 0,8/100.000 em 1974 e 1,1/100.000 em 1984.

Embora a distribuição da tuberculose para os diferentes países seja dificilmente comparável ${ }^{28.29}$, existem diferenças reais entre regiões menos desenvolvidas e mais desenvolvidas.

Verifica-se, também, que a incidência maior encontrada nesta pesquisa, na faixa etária de menores de 5 anos, difere daquela observada em alguns países, onde a transmissão provavelmente se faz fora do micro-ambiente de vida da criança, ocorrendo na fase em que ela começa contatos com outros ambientes, principalmente escolas.

Pelos dados da presente pesquisa pode-se supor a existência de infecção em micro-ambiente, facilitando a transmissão na faixa etária de menores de 5 anos.

Em relação ao sexo (Tabela 1), existem diferenças entre muitos trabalhos da literatura, ora predominando no sexo masculino ${ }^{2.7,39}$ ora no feminino ${ }^{40}$, enquanto que outros não referem diferenças entre os $\operatorname{sexos}^{18,26}$.

No que se refere às diferenças de incidência nas várias regiões de São Paulo (Tabela 2, Figura), embora não se possa, no âmbito deste trabalho, encontrar explicações para tais diferenças, poder-seia supor que devam existir fatores ambientais responsáveis pela maior incidência de tuberculose em algumas regiões. Esse fato foi observado por Carneiro e Mota ${ }^{8}$, em Salvador, Nardy ${ }^{24}$, em São Paulo, e Fernandez e col. ${ }^{14}$, na cidade de Buenos Aires.

É difícil a comparação entre as formas de tuberculose (Tabela 3), pois diferem as classificações, tanto em outros países como no próprio Brasil, onde a classificação utilizada ${ }^{6.37}$ - pulmonar e extrapulmonar - (incluindo na forma pulmonar a pulmonar associada a outros órgãos e na extrapulmonar, a meningite associada à pulmonar) é diferente daquela adotada no trabalho do Medical Research Council ${ }^{22}$, que classifica a tuberculose em respiratória, extrarespiratória e ambas, não especificando o critério adotado. Lotte e col..$^{19}$ fazem também outra classificação, assim como Carneiro e Mota ${ }^{7}$ em Salvador.

Pelos motivos expostos, a tuberculose é melhor

* Dados obtidos do Programa Nacional contra Tuberculose. Convênio INAMPS-Ministério da Saúde, 1984.

** Calculado a partir de dados fornecidos pelo Centro de Informações de Saúde (CIS).

***Dados obtidos do Programa Nacional Contra Tuberculose. Convênio INAMPS - Ministério da Saúde, 1984. 
analisada quando distribuída por órgãos ou sistemas, como foi feito nas Tabelas 4 e 5 . Pelos dados aí expostos verifica-se que a localização pulmonar da tuberculose, se considerada isoladamente, é responsável por um coeficiente de 17,3/100.000 habitantes, mas considerada associada a outras localjzações esse coeficiente sobe para 18/100.000 habitantes.

A ocorrência de tuberculose miliar, meningite, pleural e ganglionar periférica, além de casos de tuberculose osteoarticular, reflete não só as consequiências precoces da introdução de M.tuberculosis no organismo da criança, mas também a própria patogênese da tuberculose primária ${ }^{3,17,25,35}$.

Em população, cuja cobertura vacinal é provavelmente elevada, não deveria ocorrer casos de forma grave de tuberculose, como miliar e meningite. Em virtude da prática de vacinação $B C G$ indiscriminada provavelmente muitos casos são vacinados na vigência ou pós infecção por M.tuberculosis, principalmente aqueles que convivem com fonte de infecção bacilífera.

Poderia ocorrer também falha na cobertura vacinal ou mesmo a proteçâo conferida pela vacina, sem se levar em consideração outros fatores individuais ou ambientais capazes de alterar essa proteção. Um dos aspectos a considerar é aquele referido por Tidjani e col ${ }^{36}$, quando encontrou menor proteção conferida pela vacina BCG em contatos de casos com tuberculose.

No que se refere à tuberculose pleural $(2,9 \%$ $0,6 / 100.000$ ) na forma isolada (Tabela 4) e 4,6\% ou 0,9/100.000 (Tabela 5), quando associada, está de acordo com Martins ${ }^{21}$, que refere entre as formas extrapulmonares, a tuberculose pleural, juntamente com a ganglionar periférica, como mais frequientes no país, no grupo etário de menores de 15 anos.

Quanto à tuberculose ganglionar periférica, 2,3\% ou $0.5 / 100.000$ ) quando considerada isolada e $3.1 \%$ ou $0,7 / 100.000$ quando associada (Tabelas 4 e 5), ocupa, nos vários trabalhos da literatura, uma posição de destaque entre as extrapulmonares $-55,0 \%$ no trabalho realizado na Inglaterra ${ }^{22}, 52,6 \%$ no de Rodriguez e col..$^{26}$, enquanto que Farer e col. ${ }^{13}$ referem não ser muito freqüente nos Estados Unidos da América.

A proporção de tuberculose renal de $1,8 \%$ e 0,4 / 100.000 (Tabelas 4 e 5) está concordante com a literatura, que a refere, com maior frequência, em fase posterior da vida, além dos 14 anos de idade.

Por outro lado, a proporção de tuberculose miliar e de meningite, em menores de 5 anos (Tabela 5), está de acordo com a literatura, que as refere com maior frequiência, em fase muito precoce da vida.

Embora os coeficientes revelem maior incidência de casos mais graves, em fase da vida onde a resis- tência à tuberculose é menor (menores de 5 anos): embora a menor incidência de casos, na faixa etária de 10 a 14 anos, apontem essa faixa como aquela de maior resistência à tuberculose, é discrepante o encontro dc maior incidência de tuberculose miliar, no grupo de 10 a 14 anos quando comparado ao de 5 a 9 anos.

Um fato diferente do que é usualmente referido ${ }^{25}$ encontra-se na incidência de tuberculose renal, ligeiramente superior no grupo etário de 5 a 9 anos.

$O$ coeficiente de meningite, de 1,8/100.000 em menores de 5 anos, corresponderia a um risco de infecção de $0,34 \%$ ou $0,35 \%$ se calculado a partir da regra proposta por Styblo e Sutherland ${ }^{30}$ e a proposta para o Brasil ${ }^{9}$.

Se o risco de infecção para a região sudeste do Brasil, em 1985, foi de $0.6 \%$ e. se foi assumido que a partir de 1977 a queda do risco seria da ordem de $5 \%$, em 1984 o risco seria de $0,63 \%$, mesmo valor se calculado segundo dados de Arantes e col.!, para a cidade de São Paulo (riscos de $0,7 \%$ em 1982). A incidência de meningite seria respectivamente de $3,2 / 100.000$ habitantes e 3,3/ 100.000 se o critério fosse o proposto para o Brasil $^{9}$ e o de Styblo e Sutherland ${ }^{30}$, coeficiente portanto bem maior que aquele encontrado para o Município de São Paulo. Se não ocorresse a subnotificação, como refere $\mathrm{Nardy}^{24}$, poder-se-ia pensar num possível impacto da vacinação $B C G$ sobre a meningite tuberculosa. Entretanto, em face das observações acima, fica difícil seu emprego na avaliação do risco de infecção.

Por outro lado, a comparação dos dados do exame bacteriológico com os dados da literatura, no que se refere a realização e resultado, fica às vezes difícil, não só em face dos aspectos já apresentados na introdução, bem como da diferença existente entre as classificações da tuberculose $e^{6.19 .22 .37}$.

Para ser possível comparar os dados da presente pesquisa com os dados do Estado de São Paulo e do Brasil, foi preciso fazer a correção de acordo com o critério de classificação adotado para o país ${ }^{12}$. Verifica-se, dessa forma, que a proporção de exames realizados na forma pulmonar, em 1984, no Município de São Paulo, foi bem maior do que a do Estado de São Paulo*, no grupo etário de menores de 15 anos $(20,6 \% / 11,2 \%$, respectivamente para o Município e Estado), enquanto que para o Bra sil** foi de $33,2 \%$ e no trabalho de Cameiro e $\mathrm{Mota}^{\top}$, de $50 \%$.

A proporção de exames positivos à pesquisa de BK varia muito de pesquisa a pesquisa. Nas estatísticas do Estado de São Paulo* e do Brasil** as proporções de positivos foram, respectivamente, $48.9 \%$ e $58,7 \%$ em menores de 15 anos, enquanto que Carneiro e Mota ${ }^{7}$ encontraram 30,8\% de exames positivos. 
$\mathrm{Na}$ pesquisa realizada pelo Medical Research Council $^{22}$ a proporção de positivos foi de $39,0 \%$ entre os exames realizados $(6,0 \%$ ao exame bacterioscópico), o que corresponde a $14 \%$ de crianças com doença respiratória, enquanto que no trabalho posterior, da mesma entidade, em $1983^{23}$, os resultados mostram que houve confirmação bacteriológica em $19 \%$ de crianças com tuberculose respiratória, não havendo referência à proporção de positivos entre os exames realizados. Lotte e col..$^{19}$ encontraram proporção de positivos de $15,3 \%$, considerando-se o exame direto e a cultura, na forma respiratória.

Essas considerações mostram as diferenças existentes entre as pesquisas, tornando difícil a comparação dos respectivos coeficientes, que são influenciados pelo número de casos em que o exame é realizado e a proporção de positivos entre eles.

Por outro lado, nos trabalhos da literatura, o exame bacteriológico ora aparece como resultado de baciloscopia de escarro, ora englobando tanto a baciloscopia como a cultura em material que pode ser escarro, lavado gástrico ou outros.

Os coeficientes de incidência de casos positivos à pesquisa de $\mathrm{BK}$ no Estado de São Paulo* foram 0,$3 ; 0,2 ; 2,5$ e $0,9 / 100.000$, respectivamente para menores de 5 anos, 5 a 9 anos 10 a 14 e menores de 15 anos, enquanto que no Brasil** as taxas foram $1,2 / 100.000$ em menores de 5 anos, 1,8/100.000 para 5 a 9 anos e $5,4 / 100.000$ para 10 a 14 anos, com uma taxa total de 2,6/100.000 em menores de 15 anos.

$\mathrm{Na}$ Argentina ${ }^{20}$ os coeficientes foram 2,5; $2,7 \mathrm{e}$ $8,9 / 100.000$ para menores de $5 ; 5$ a 9 e 10 a 14 anos, para casos confirmados bacteriologicamente. Irurzun e col. ${ }^{15,16}$, em Buenos Aires, referem, para 1984, coeficientes de 0,5 e 1,7/100.000, respcetivamente para menores de 5 e no grupo etário de 5 a 14 anos para casos confirmados bacteriologicamente, o que corresponde, no Município de São Paulo, a 1,2 e 1,7/100.000, respectivamente para os mesmos grupos etários.

Do ponto de vista do coeficiente de bacilíferos, representado pelos casos positivos à baciloscopia de escarrro (casos de tuberculose do ponto de vista epidemiológico), verifica-se que é muito pequeno no grupo etário de menores de 15 anos (Tabela 7), sendo que o grupo de 10 a 14 concorre com o maior coeficiente. Por esses dados verifica-se que a criança seria um elo muito pequeno na cadeia

* Calculado a partir de dados fornecidos pelo Centro de Informaçōes da Saúde (CIS).

** Dados obtidos do Programa Nacional Contra Tuberculose. Convênio INAMPS - Ministério da Saúde, 1984. epidemiológica de transmissão, mesmo na faixa etária de 10 a 14 anos.

\section{Agradecimento}

Ao Prof. Dr. Roberto Brólio, pelas valiosas sugestões e apoio à realização do presente trabalho.

DIAS, M.H. de P. [Epidemiological aspects of tuberculosis for minors under 15 years old in a County of the Brazilian Southeast Region, 1984. Rev. Saúde públ., S.Paulo, 25: 426-34, 1991. Reported cases of tuberculosis in minors under fifteen years old in S.Paulo County, State of S.Paulo, Brazil, are analyzed. The Health Information Center of the Health Secretariat of the State of S. Paulo was notified of the diagnosis in all cases which occurred in 1984. The medical records were analyzed and the physicians in charge of the reports interviewed. Among the population studied the annual notification rate was $21.4 / 100,000$ for minors under fifteen with largest incidence $(31.8 / 100,000)$ for those under fifteen years old. Significant discrepancies between the various regions were observed. Pulmonary tuberculosis was by far the most predominant form of the disease $(83.1 \%$ and 17.8 / 100,000 ). Pulmonary tuberculosis associated with extrapulmonary cases accounts for $4.7 \%$ of all the cases $(1.100,000)$. Extrapulmonary cases $(2.5 / 100,000)$ accounted for $12.2 \%$ of all reports. Also, a significant coefficient $(1 / 100,000)$ was observed for meningitis (isolated or associated), equivalent to $4.9 \%$ of all cases. Nearly $35.7 \%$ (46 cases) of the lung material analysed revealed $\mathrm{BK}+$. Of the extrapulmonary material analysed sixteen cases $(53.3 \%)$ of them were BK+. The incidence of positive sputum smear cases was $0.9 / 100,000$ inhab., being greater in the age group 10-14 (2.8/100,000 inhab).

Keywords: Tuberculosis in childhood, epidemiology. Disease notification.

\section{Referências Bibliográficas}

1. ARANTES, G.R. et al. Estudo sobre a evolução do risco de infecção tuberculosa em área com elevada cobertura por BCG. Rev. Saúde públ., S.Paulo, 19:95-107, 1985.

2. BAHL, L. et al. A study on tuberculosis in children in and around Simla Hiłls. J. Indian med. Ass., 82:44-7, 1984.

3. BROLIO, R. \& LIMA FILHO. M.T. Tuberculose pulmonar. In: Veronesi, R., ed. Doenças infecciosas e parasitarias. $7^{\text {a. }}$ ed. Rio de Janeiro, Guanabara Koogan, 1982. p.314-33.

4. BULLA, A. Rapport de statistiques sanitaires mondiales. Tuberculose: combien de malades? Bull. Un. int. Tuberc.,52:39-44, 1977.

5. BULLA, A. La tuberculosis en el mundo. I. Revision de la morbilidad y de la m ortalidad por tuberculosis en el mundo (1967-1971-1977), basada en las informaciones oficiales. Bol.Un. int. Tuberc.56: 121-7, 1981.

6. CAPORALI, M.L. Organização do arquivo de informaçôes sobre tuberculose do Estado de São Paulo. São Paulo, 1982. [Dissertação de Mestrado - Faculdade de Saúde Pública da USP].

7. CARNEIRO, N.M.B. \& MOTA, E. Tuberculose em Salvador: características clínico-epidemiológicas em 1980. Rev. baia. Saude públ., 13: 21-32, 1986.

8. CARNEIRO, N.M.B. \& MOTA, E. Tuberculose em Salvador Bahia: incidência e algumas variáveis sócio-demográficas em 1980. Rev. baia. Saúde públ., 13: 68-80, 1986. 
9. CONTROLE da Tuberculose: uma promessa de integração Ensino-Serviço. Brasília, Campanha Nacional Contra a Tuberculose/Funcação Universitária José Bonifácio UFRJ, 1987.

10. COSTA, D.C. Considerações sobre a tendência da tuberculose no Brasil. Cad. Saúde públ., Rio de Janeiro, 1:313-26, 1985.

11. DAVIES, P.D.O. et. al. Ambiguities and inaccuracies in the notification system for tuberculosis in England and Wales. Community Med., 3: 108-18, 1981.

12. DIAS, M.H.P. Aspectos epidemiológicos da tuberculose em menores de 15 anos, no Município de São Paulo, 1984. São Paulo, 1989. [Tese de Doutoramento - Faculdade de Saúde Pública da USP]

13. FARER, L.S. et al. Extrapulmonary tuberculosis in the United States. Amer. J. Epidem., 109: 205-17, 1979.

14. FERNANDEZ, G. et. al. Los determinantes sociales de la tuberculosis en la ciudad de Buenos Aires. Med. y Soc.,7: 64-70, 1984.

15. IRURZUN, R.N. et. al. Percepcion de morbilidad y atencion medica en tuberculosis. Med. y Soc., 9: 66-72, 1986.

16. IRURZUN, R.N. et. al. Epidemiologia y control de la tuberculosis en la ciudad de Buenos Aires. Rev, argent. Tuberc.,48: 17-34, 1987.

17. LINCOLN, E.M. \& SEWELL, E.M. Tuberculosis in children. New York, Mc Graw-Hill, 1963.

18. LÓPEZ, C.V. \& SOUZA, M. Evolucion de la tuberculosis en el hospital del niño en 10 anos. Rev. Hosp. Niño, Panama, 5: $11-7,1985$.

19. LOTTE, A. et. al. Epidemiologie de la tuberculose et défaillances de la lutte anti tuberculose chez l'enfant. Bull. Wld Hlth Org., 44: (Suppl.), 1971.

20. MARCHESE, A.F. Morbilidad por tuberculosis en la Republica Argentina. Rev. argent. Tuberc., 47: 69-71, 1986.

21. MARTINS, S. Epidemiologia da tuberculose. In: Sant'Anna C.C. \& Bethlem, N. Tuberculose na infância. $2^{a}$. ed. Rio de Janeiro, Ed. Cultura Médica, 1988. p. 4-9.

22. MEDICAL RESEARCH COUNCIL TUBERCULOSIS AND CHEST DISEASES. Tuberculosis in children in a national survey of notifications in England and Wales, 1978-1979. Arch. Dis. Child.,57: 734-41, 1982.

23. MEDICAL RESEARCH COUNCIL TUBERCULOSIS AND CHEST DISEASES. Tuberculosis in children in a nationa survey of notification in England and Wales in 1983. Arch. Dis. Child.,63: 266-76, 1988.

24. NARDY, S.M.C. Contribuição para o estudo epidemiológico da meningite tuberculosa na Grande São Paulo. São Paulo, 1986. [Tese de Doutoramento - Faculdade de Saúde Pública da USP].

25. RICH, A.R. Patogenia de la tuberculosis. Buenos Aires, Editorial "Alfa", 1946.
26. RODRIGUEZ, L.E.S. et. al. Tuberculosis en el niño: presentacion de 114 casos. Rev. med. Hosp. gen., México, 45: $219-26,1982$.

27. ROMANUS, V. Tuberculosis in Bacillus Camette Guérin/ immunized and unimmunized children in Sweden: a ten year evaluation following the cessation of general Bacillus Calmette-Guerin immunization of the newborn in 1975. Pediat. infect. Dis., 6: 272-80, 1987.

28. STYBLO, K. Estado del arte. I-Epidemiologia de la tuberculosis. Bol. Un. int. Tuberc., 53: 145-57, 1978

29. STYBLO, K. \& ROUILLON, A. El presente, y el porvenir. II. Estimaciones de la incidência mundial de la tuberculosis con baciloscopia positiva. No fiabilidad de las cifras de tuberculosis informadas oficialmente. Bol. Un. int. Tuberc., 56: 128-37, 1981.

30. STYBLO, K. \& SUTHERLAND, I. Epidemiological indices for planning, surveillance and evaluation of tuberculosis programmes. Bull. int. Un. Tuberc., 49: $66-73,1974$.

31. STYBLO, K. \& SUTHERLAND, I. El presente y el porvenir. IV. Epidemiologia de la tuberculosis infantil. Bol. Un. int. Tuberc., 57: 134-41, 1982.

32. STYBLO, K. et. al. The transmission of tubercle bacilli. It's trend in a human population. Bull. int. Un. Tuberc.,42, Mar., 1969.

33. SUMÁRIO DE DADOS DA GRANDE SÃO PAULO. São Paulo, Empresa Metropolitana de Planejamento da Grande São Paulo, 1985.

34. SUTHERLAND, I. Recent studies in the epidemiology of tuberculosis based on risk of being infected with tubercle bacilli. Adv. Tuberc. Res., 19: 1-63, 1976.

35. TARANTINO, A.B. et al. Tuberculose. In: Tarantino, A.B. Doenças pulmonares. Rio de Janeiro, Ed. Guanabara Koogan, 1976. p. 329-98.

36. TIDJANI, $O$. et al. The protective effect of $B C G$ vaccination of the newborn against childhood tuberculosis in an African Community. Tubercle, 67: 269-81, 1986.

37. TUBERCULOSE. Radis-Tema FIOCRUZ, dez., 1982.

38. TUBERCULOSIS control. Tokyo, Research Institute of Tuberculosis. Japan Anti-Tuberculosis Association/World Health Organization, 1979. (WHO-Japan International Tuberculosis Course).

39. WEILAND, P. Die heutige Tuberkulose Situation Bei Kindern und Jugendlichen in der DDR. Z. Erkrank. Atm. Org., 164: $150-4,1985$.

40. WILSON, J.M. et al. Tuberculosis in Eskimo children. Amer Rev. resp. Dis, 108: 559-64, 1973.

Recebido para publicação em 11/01/1991. Reapresentado em 01/08/1991. Aprovado para publicação em 24/08/1991. 